\title{
NS3 Variability in Hepatitis C Virus Genotype 1 A Isolates from Liver Tissue and Serum Samples of Treatment-Naïve Patients with Chronic Hepatitis C
}

\author{
Deborah D'Aliberti ${ }^{a, b}$ Irene Cacciola ${ }^{a, b}$ Cristina Musolino ${ }^{a, b}$ \\ Giuseppina Raffa ${ }^{a}$ b Roberto Filomia ${ }^{a, b}$ Angela Alibrandic \\ Salvatore Benfatto ${ }^{d}$ Concetta Beninati ${ }^{d}$ Carlo Saitta $^{a} b$ Domenico Giosa ${ }^{e}$ \\ Orazio Romeo $^{\text {e,f }}$ Giovanni Raimondo ${ }^{a, b}$ Teresa Pollicino ${ }^{b, d}$

\begin{abstract}
${ }^{a}$ Department of Clinical and Experimental Medicine, University Hospital of Messina, Messina, Italy; ${ }^{b}$ Division of Clinical and Molecular Hepatology, University Hospital of Messina, Messina, Italy; ${ }^{C}$ Department of Economics, University of Messina, Messina, Italy; ${ }^{\text {d}}$ Department of Human Pathology, University Hospital of Messina, Messina,

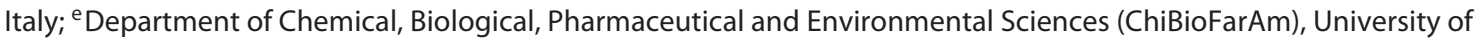
Messina, Messina, Italy; ${ }^{f}$ IRCCS Centro Neurolesi Bonino-Pulejo, Messina, Italy
\end{abstract}

\section{Keywords}

Hepatitis C virus · Chronic hepatitis C · Genotype-1a

\begin{abstract}
Background: Hepatitis C virus (HCV) NS3 resistance-associated substitutions (RASs) reduce HCV susceptibility to protease inhibitors. Little is known about NS3 RASs in viral isolates from the liver of chronic hepatitis $\mathrm{C}(\mathrm{CHC})$ patients infected with HCV genotype-1a (G1a). Aim: The objective of this work was to study NS3 variability in isolates from the serum and liver of HCV-G1a-infected patients naïve to direct-acting antivirals (DAAs). Methods: NS3 variability of HCV-G1a isolates from the serum and liver of 11 naïve $\mathrm{CHC}$ patients, and from sera of an additional 20 naïve CHC patients, was investigated by nextgeneration sequencing. Results: At a cutoff of $1 \%$, NS3 RASs were detected in all the samples examined. At a cutoff of $15 \%$, they were found in $54.5 \%(6 / 11)$ and $27.3 \%(3 / 11)$ of the
\end{abstract}

\section{KARGER}

(c) 2018 S. Karger AG, Basel

E-Mail karger@karger.com

www.karger.com/int paired liver and serum samples, respectively, and in $22.5 \%$ (7/31) of the overall serum samples examined. Twenty-six out of thirty-one (84\%) patients showed NS3 variants with multiple RASs. Phylogenetic analysis showed that NS3 sequences clustered within 2 clades, with 10/31 (32.2\%) patients infected by clade I, $15 / 31$ (48.8\%) by clade II, and 6/31 (19.3\%) by both clades. Conclusions: Though the number of patients examined was limited, NS3 variants with RASs appear to be major components of both intrahepatic and circulating viral quasispecies populations in DAA-naïve patients.

() 2018 S. Karger AG, Basel

\section{Introduction}

Hepatitis $\mathrm{C}$ virus (HCV) infection is a serious health problem worldwide. Currently, approximately 71 million people, representing $1 \%$ of the world's population, are 
Table 1. Demographic and clinical characteristics of the studied patients

\begin{tabular}{|c|c|c|c|}
\hline & $\begin{array}{l}\text { With liver biopsy } \\
(n=11)\end{array}$ & $\begin{array}{l}\text { Without liver biopsy } \\
(n=20)\end{array}$ & $p$ value \\
\hline Age, years & $50(45-55)$ & $56(43-60)$ & 0.1 \\
\hline Male gender, $n$ & $8(72)$ & $13(65)$ & 0.1 \\
\hline Median HCV RNA level, $\log _{10} \mathrm{IU} / \mathrm{mL}$ & $6.01(5.65-6.44)$ & $5.90(5.25-6.08)$ & 0.8 \\
\hline Chronic hepatitis/cirrhosis, $n$ & $4 / 7^{\mathrm{a}}$ & $11 / 10^{\mathrm{b}}$ & 0.4 \\
\hline \multicolumn{4}{|l|}{ DAA treatments, $n$} \\
\hline Including PIs & $5(45.5)$ & $11(55)$ & 0.7 \\
\hline Not including PIs & $6(54.5)$ & $9(45)$ & 0.6 \\
\hline History of intravenous drug abuse & $6(54.5)$ & $11(55)$ & 1 \\
\hline
\end{tabular}

chronically infected with HCV, and more than 399,000 people die from HCV-related liver diseases each year [1]. Recently, several highly potent direct-acting antivirals (DAAs) have been developed for the treatment of chronic hepatitis $\mathrm{C}(\mathrm{CHC})$, and 4 classes of DAAs targeting specific key components of viral replication, such as HCV NS3/4A, HCV NS5A, and HCV NS5B proteins, have so far been licensed for $\mathrm{CHC}$ treatment $[2,3]$. However, treatment of $\mathrm{HCV}$ infection with DAA is associated with very high rates of sustained virologic response, and viral variants with resistance-associated substitutions (RASs) conferring reduced susceptibility to DAAs may emerge during antiviral therapy, this event being one of the main factors responsible for the few but existing cases of virologic treatment failure [4-6]. These naturally occurring resistant variants can emerge and become the dominant viral infecting population under the selective pressure of an antiviral drug and when complete suppression of viral replication is not achieved [7]. Thus, investigation of $\mathrm{HCV}$ variability in the genomic regions targeted by DAAs is of key importance if the goal of extensive treatment is to reach $100 \%$ of therapeutic success with the possible elimination of the virus at least from some geographic areas. HCV genomic heterogeneity has been evaluated in numerous studies and some important information is available [4-6]. In fact, emergence and takeover of variants carrying NS3 polymorphisms associated with protease inhibitors (PIs) resistance appears to be a quite frequent occurrence, particularly in patients infected with HCV genotype 1, subtype a (G1a), thus partially limiting the use of some PIs (i.e., simeprevir) in these cases [4-6].
The frequency of naturally occurring G1 NS3-resistant variants has been reported to range between 0.1 and $3.1 \%$, with the exception of the Q80K variant that has a good replicative fitness and is present from 19 to $48 \%$ of isolates belonging to clade I of HCV Gla, which is reported to be typically diffused in North America [5, 6, 8-10].

NS3 polymorphisms of HCV G1a potentially conferring resistance to PIs have been investigated in HCV isolates from the serum of patients, whereas - apart from anecdotic cases [11-13] - no extensive evaluation of isolates from the liver that is the site of virus replication and where $\mathrm{HCV}$ quasispecies diversity reaches the highest level of complexity $[11,12,14-16]$ has been performed so far. In addition, almost all the studies have evaluated the presence of RASs by performing population sequencing, which is known to have a quite low sensitivity (15-20\% frequency within the HCV quasispecies) to detect viral variants $[4,17]$. In this study, we used next-generation sequencing (NGS) to investigate the prevalence of $\mathrm{HCV}$ NS3 amino acid (aa) substitutions in viral isolates from serum and liver samples of patients from Southern Italy infected with HCV Gla and naïve to any DAA treatment.

\section{Patients and Methods}

Patients

Serum samples from 31 naïve patients (21 males and 10 females with a median age of 54 years, range $43-60$ years; Table 1) with HCV-Gla chronic infection consecutively attending the Liver Unit of the Messina University Hospital were collected from January 2010 to December 2015 and stored at $-80^{\circ} \mathrm{C}$. Tissue samples
D’Aliberti et al. 
Table 2. HCV-specific oligonucleotide sequences used for amplification and sequencing of NS3 protease

\begin{tabular}{llll}
\hline Name & Polarity & Sequence primer $\left(5^{\prime} \rightarrow 3^{\prime}\right)$ & Position HCV H77, bp \\
\hline OUT NS3F & Forward & CGCGTGCGGTGACATCATC & $2970-2989$ \\
OUTNS3 R & Reverse & AGCACYAACGTSCCGGCTG & $3705-3724$ \\
NESTED-NS3 MiS I F & Forward & *Adapter1-ACWGGCCGGGACAARAACCA & $3139-3159$ \\
NESTED-NS3 MiS I R & Reverse & *Adapter2-TCCTACYTGAARGGCTCYTCGG & $3472-3494$ \\
NESTED-NS3 MiS II F & Forward & *Adapter1-CTCRGACCTTAYYTGGTCA & $3384-3403$ \\
NESTED-NS3 MiS II R & Reverse & *Adapter2-CYCCGGTSTTCACRGAYAACT & $3613-3634$ \\
\hline
\end{tabular}

$\mathrm{Y}=\mathrm{C}$ or $\mathrm{T}, \mathrm{S}=\mathrm{G}$ or $\mathrm{C}, \mathrm{W}=\mathrm{A}$ or $\mathrm{T}, \mathrm{R}=\mathrm{A}$ or $\mathrm{G}$ according to IUPAC nucleotide code. bp, base pair. ${ }^{*}$ Adapter $(1$ or 2$)$ primer sequences not shown (Illumina technology).

obtained by percutaneous needle biopsy at the time of blood sampling, immediately frozen in liquid nitrogen and stored at $-80^{\circ} \mathrm{C}$, were also available from 11 of the 31 patients included in the study. Written informed consent was obtained from each patient. The study protocol conformed to the principles of the Declaration of Helsinki and was reviewed and approved by the local Ethics Committee.

Serum HCV RNA levels were quantified in each sample using the commercial assays COBAS Ampliprep/COBAS TaqMan HCV Quantitative Test v2.0 (Roche Diagnostics, Mannheim, Germany) with a lower limit of detection of $15 \mathrm{IU} / \mathrm{mL}$. HCV genotype/subtypes were determined at screening by Versant LIPA v2 assay (Siemens Healthcare Diagnostics, Milan, Italy) and also confirmed by sequencing analysis of the NS5A, NS5B, and NS3/4A viral regions followed by alignment search tool (Geno2pheno) analysis [18].

\section{NS3 Amplification, Library Preparation, and NGS}

HCV RNA was extracted from serum and liver tissue samples using the PureLink Viral RNA/DNA Mini Kit (Invitrogen-Life Technologies, Paisley, UK) and RNeasy Micro Kit (Qiagen, Hilden, Germany), respectively. Complementary DNA was synthesized from RNA extracts using the Transcriptor First Strand cDNA Synthesis Kit (Roche Diagnostics) and random hexamers, according to the manufacturer's instructions. The entire NS3-coding region was amplified by nested polymerase chain reaction (PCR) using the Expand High Fidelity PCR System (Roche Diagnostics) and $\mathrm{HCV}$ gene-specific primers. Primers used in the second round of nested PCR contained a barcode sequence unique to each sample and the Illumina MiSeq flow cell adaptors. The primers used for amplification and sequencing are reported in Table 2. PCR experiments were performed using a $50-\mu \mathrm{L}$ reaction volume containing $5 \mu \mathrm{L}$ of $10 \mathrm{X}$ Taq Buffer, $4 \mu \mathrm{L}$ of $25 \mathrm{mM} \mathrm{Mg}^{2+}, 300 \mathrm{nM}$ of each primer, $200 \mu \mathrm{M}$ of each of the 4 dNTPs, and 2.6 units of the Expand High Fidelity DNA Polymerase. The conditions for the first round of nested PCR included: an initial denaturing step at $94^{\circ} \mathrm{C}$ for $4 \mathrm{~min}$, followed by 35 cycles of a denaturing step at $94^{\circ} \mathrm{C}$ for $30 \mathrm{~s}$, an annealing step at $60^{\circ} \mathrm{C}$ for $50 \mathrm{~s}$, an extension step at $72^{\circ} \mathrm{C}$ for $1 \mathrm{~min}$, then followed by a final extension at $72^{\circ} \mathrm{C}$ for $10 \mathrm{~min}$. Products of the first round PCR then underwent a second round of processing consisting of the steps of first PCR but with an annealing temperature decrease to $56^{\circ} \mathrm{C}$. The final PCR product was detected by agarose gel (1.5\%) electrophoresis. PCR products were purified using the QIAquick PCR Purification Kit (Qiagen), and then quantified using the Quant-It dsDNA High Sensitivity Kit and the Qubit 2.0 fluorometer (Life Technologies, Paisley, UK). The purified PCR products were converted to Illumina sequencing libraries using the Nextera XT Sample Preparation KIT (Illumina, Litte Chesterford, UK) and the Nextera XT Index Kit (Illumina). Libraries were pooled and spiked with 5\% Phix Control Kit v3 (Illumina), according to the manufacturer's instructions. Sequencing was performed on the Illumina MiSeq using $2 \times 250$ cycles v 2 chemistry (Illumina Inc., San Diego, CA, USA). Illumina sequencing raw reads $(4,903,637$ in total) were processed using Trimmomatic ( $v 0.36$ ) [19] to remove adapters and sequences with low Phred-scores (cutoff $\geq 30$, minimum read length $35 \mathrm{bp}$ ). After quality filtering and trimming, approximately $4 \%$ of the total reads were removed, leaving a large data set $(4,714,691$ in total) of high-quality reads for subsequent genotyping analysis. For consensus sequence reconstruction, reads from each patient were aligned against a large dataset of reference clade I/clade II sequences (online suppl. Table 1; see www.karger.com/doi/10.1159/000489307 for all online suppl. material) using BWA (with the option - $\mathrm{x}$ intractg) [20] and further analyzed by the BLAST+ program [21].

A threshold value $\geq 70 \%$ (percentage of the total reads allocated to a single reference sequence) was used to delineate a distinct consensus sequence belonging exclusively to the clade I or clade II genotype (online suppl. Table 1). Samples with mapping values below this threshold (3B, 3S, 7B, 9B, 12S, 27S and 31S) were considered as mixed samples and 2 different consensus sequences were retrieved (clade I and clade II). Consensus sequences were called by combining SUPER-CAP [22] and SAMtools programs [23], and used in MEGA 7 software [24] to create a maximum likelihood phylogenetic tree (Fig. 1) based on the general time reversible substitution model (GTR+G+I option) which was selected by best model search using the "Find best DNA Model" function implemented in MEGA7 [24]. RASs were analyzed using 1, 5, 10, 15, and $20 \%$ cutoffs.

\section{Statistics}

Continuous numerical variables are expressed as the median and interquartile range and categorical variables as the number and percentage. For each RAS, absolute frequencies and percentages were calculated for both serum and tissue samples.

Examined continuous variables did not present a normal distribution as verified by the Kolmogorov-Smirnov test and the nonparametric approach was consequently used. To evaluate the asso- 


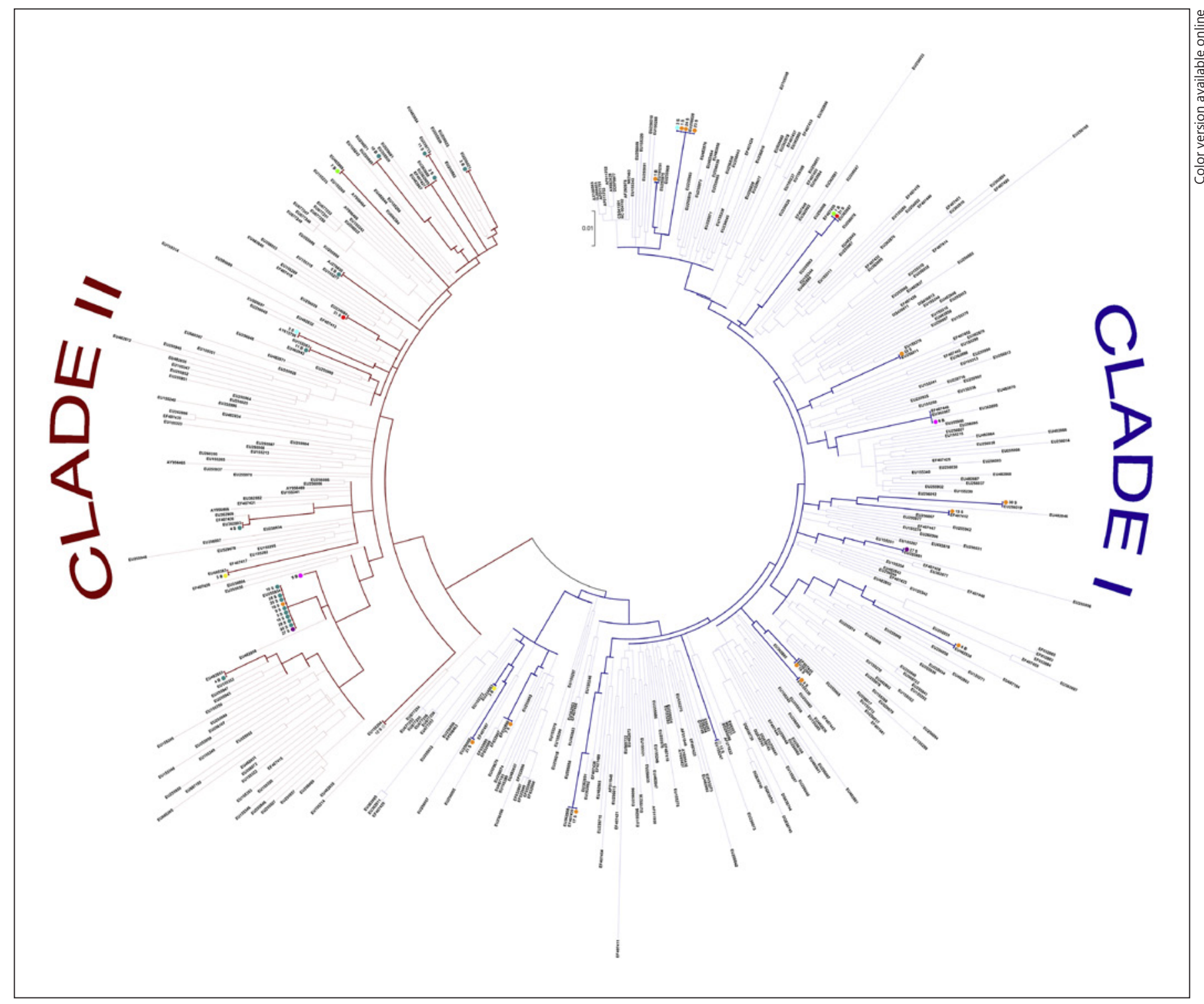

Fig. 1. Phylogenetic tree showing evolutionary relationships between our HCV subtype la sequences and reference isolates included in this study. The evolutionary history was inferred using the maximum likelihood method based on the GTR+G+I as the best-fit model of evolution. Our viral isolates are highlighted with colored circles: red (31S); yellow (3B); pink (9B); blue (12S); light

ciation between mutations, we applied contingency tables ( $\chi^{2}$ test or Fisher exact test if frequencies less than 5 were expected). The Mann-Whitney $U$ test was used to compare HCV-RNA values in $\mathrm{HCV}$-positive patients with or without liver biopsy. The Student $t$ test was used to compare normally distributed continuous variables.

Statistical analysis was performed using SPSS 17.0 for Windows (SPSS Inc., Chicago, IL, USA). $p$ values were calculated with 2 -tailed statistical analysis and $p$ values $<0.05$ were considered to be statistically significant. blue (3S); green (7B); violet (27S); teal (2B, $2 \mathrm{~S}, 4 \mathrm{~B}, 4 \mathrm{~S}, 5 \mathrm{~B}, 5 \mathrm{~S}, 8 \mathrm{~B}$, 8S, 9S, 10B, 10S, 11B, 11S, 14S, 15S, 16S, 19S, 20S, 25S, 26S, 28S, and $29 \mathrm{~S})$; orange (1B, 1S, 6B, 6S, 7S, 13S, 17S, 18S, 21S, 22S, 23S, $24 \mathrm{~S}$, and $30 \mathrm{~S})$. GenBank accession numbers for reference clade I and clade II isolates are also shown.

\section{Results}

Demographic and clinical characteristics were comparable between the 11 patients from whom both serum and liver samples were available and the 20 patients from whom only serum samples were available (Table 1). All the 31 studied patients were subsequently treated with interferon-free DAA-based regimens (13 including and 


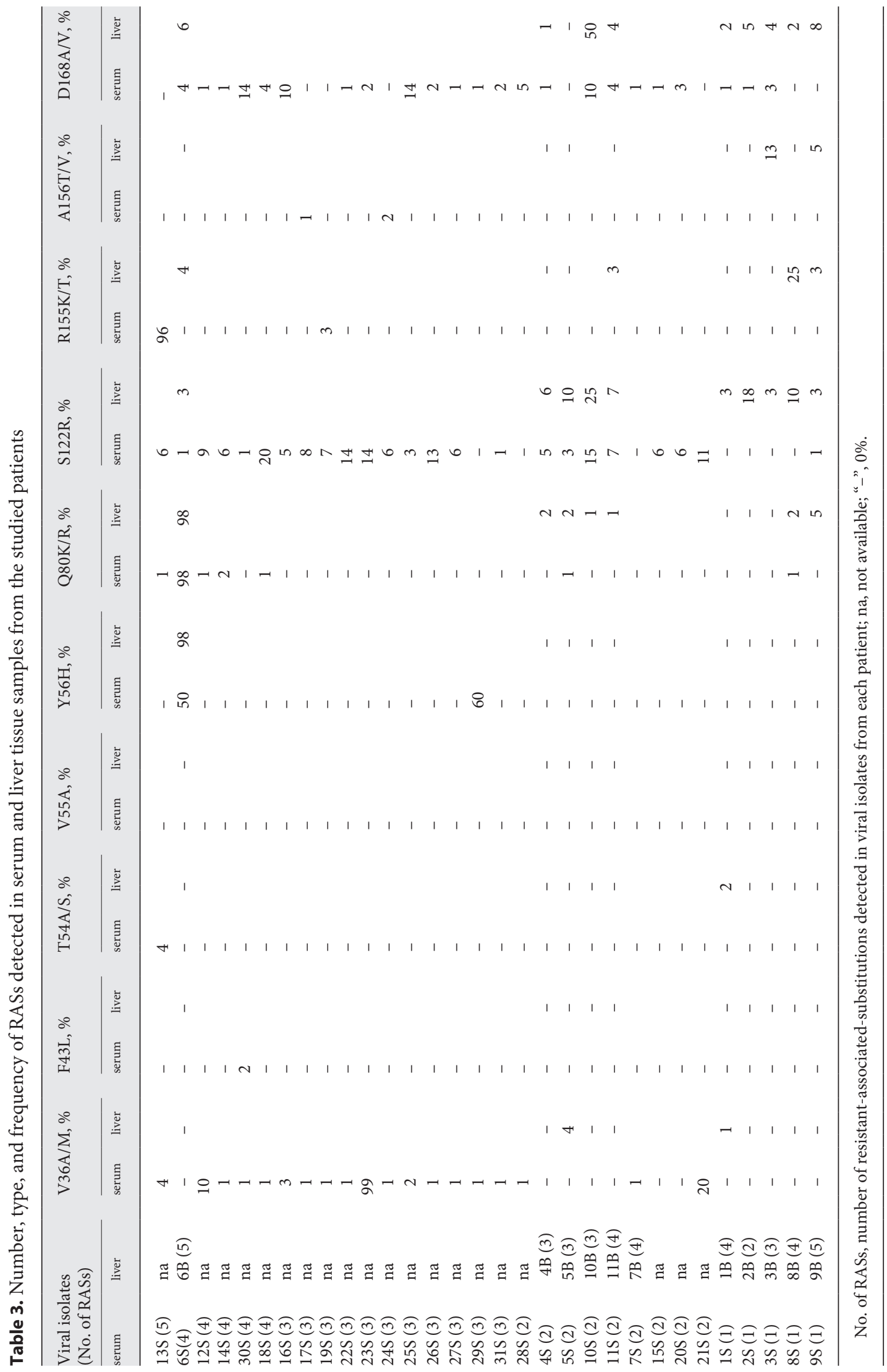


18 not including PIs) and all of them showed a sustained virologic response.

NS3 deep sequencing was successful for all the liver specimens and serum samples examined. The analysis of polymorphisms at residues associated with resistance to PIs (including substitutions at position V36, F43, T54, V55, Y56, Q80, S122, R155, A156, and D168 of the NS3 protease gene) [4-6] in the study population revealed that $4 / 31$ individuals (13\%) had dominant resistance variants (at a frequency of 60-99\%), conferring resistance to PIs (Table 3).

At the clinically relevant cutoff of $15 \%$ [4], the percentage of patients with baseline NS3 RASs was $54.5 \%(6 / 11$ patients) and $27.3 \%$ (3/11 patients; $p=0.2)$ when the paired liver tissue and serum samples, respectively, were evaluated, and $22.5 \%(7 / 31)$ when the sera from all the studied patients were analyzed. However, at a $1 \%$ cutoff, all the patients showed baseline NS3 RASs both in liver tissues and serum samples. In addition, at $1 \%$, cutoff, the most frequent RASs present in liver tissue specimens and paired serum samples from the 11 patients were $\mathrm{S} 122 \mathrm{R}>\mathrm{D} 168 \mathrm{~A} /$ $\mathrm{V}>\mathrm{Q} 80 \mathrm{~K} / \mathrm{R}>\mathrm{R} 155 \mathrm{~K} / \mathrm{T}>\mathrm{V} 36 \mathrm{~A}>\mathrm{A} 156 \mathrm{~T}>\mathrm{Y} 56 \mathrm{H}=\mathrm{T} 54 \mathrm{~A}$ and $\mathrm{D} 168 \mathrm{~A} / \mathrm{V}>\mathrm{S} 122 \mathrm{R}>\mathrm{Q} 80 \mathrm{~K} / \mathrm{R}>\mathrm{Y} 56 \mathrm{H}=\mathrm{V} 36 \mathrm{~A}$, respectively; at $15 \%$ cutoff they were S122R $>$ V36A $=\mathrm{Y} 56 \mathrm{H}=\mathrm{Q} 80 \mathrm{~K}=$ $\mathrm{R} 155 \mathrm{~K}=\mathrm{D} 168 \mathrm{~V}$ and $\mathrm{Y} 56 \mathrm{H}=\mathrm{Q} 80 \mathrm{~K}=\mathrm{S} 122 \mathrm{R}$, respectively. When serum samples from all the 31 patients were evaluated, the RASs most frequently found at $1 \%$ cutoff were $\mathrm{S} 122 \mathrm{R}>\mathrm{D} 168 \mathrm{~A} / \mathrm{V}>\mathrm{V} 36 \mathrm{~A}>\mathrm{Q} 80 \mathrm{~K} / \mathrm{R}>\mathrm{Y} 56 \mathrm{H}=\mathrm{R} 155 \mathrm{~K} / \mathrm{T}=$ $\mathrm{A} 156 \mathrm{~V}>\mathrm{F} 43 \mathrm{~L}=\mathrm{T} 54 \mathrm{~A}$, and at $15 \%$ cutoff were $\mathrm{V} 36 \mathrm{~A}=$ $\mathrm{Y} 56 \mathrm{H}=\mathrm{S} 122 \mathrm{R}>\mathrm{Q} 80 \mathrm{~K}=\mathrm{R} 155 \mathrm{~K}$.

At $1 \%$ cutoff, S122R and D168V/A were the most frequently detected polymorphisms both in the paired liver tissues (found in $11 / 11$ patients, $100 \%$, and $10 / 11,90.9 \%$, respectively) and serum samples (found in 6/11 patients, $54.5 \%$, and $8 / 11,72.7 \%$, respectively), as well as in serum samples from all the 31 studied patients (found in 24/31 patients, $77.4 \%$, and $23 / 31,74.2 \%$, respectively). Q80K/R was the third most frequent polymorphism found using the $1 \%$ cutoff $(8 / 11,72.7 \%$, and $3 / 11,27.3 \%$, of the cases when the paired liver and serum samples were analyzed, respectively, $p=0.03$ ). Overall, this polymorphism was detected in 7 of the 31 serum samples examined (22.6\%). In all but 1 case, the Q80K/R substitution was detected in combination with other RASs. In particular, when a cutoff of $1 \%$ was used it was significantly associated with the R155K/T substitution ( $p=0.01$ ), whereas at a cutoff of $15 \%$ it was significantly associated with the $\mathrm{Y} 56 \mathrm{H}$ substitution $(p=0.01)$. R155K/T was found in 36.4 and $6.4 \%$ of the cases when liver tissue specimens and serum samples were analyzed, respectively. The R155K/T substitution in combination with $\mathrm{Q} 80 \mathrm{~K} / \mathrm{R}$ could be detected at a frequen- cy ranging from 3 to $96 \%$ in the intrapatient HCV populations. $\mathrm{Y} 56 \mathrm{H}$ was found in 9.1 and $6.5 \%$ of the cases when liver specimens and serum samples were analyzed, respectively. The $\mathrm{Y} 56 \mathrm{H}$ substitution in combination with $\mathrm{Q} 80 \mathrm{~K} / \mathrm{R}$ could be detected at a frequency ranging from 50 to $98 \%$. Of note, the Q80K substitution was detected at a very high frequency (98\%) both in the liver and in the serum from only 1 patient. It is noteworthy that this patient showed a combination of different RASs. Indeed, besides the Q80K substitution, the $\mathrm{Y} 56 \mathrm{H}$ (present at a frequency of $98 \%$ in the liver and $50 \%$ in the serum), the S122R (present at a frequency of $3 \%$ in the liver and $1 \%$ in the serum), and the $\mathrm{D} 168 \mathrm{~V}$ (present at a frequency of $6 \%$ in the liver and $4 \%$ in the serum) substitutions could also be detected.

Importantly, the A156T substitution, known to confer high levels of resistance [4], was detected in the liver tissue specimens from 2 cases. In 1 case, the A156T RAS (present at a frequency of 13\%) was detected in combination with the S122R (present at a frequency of 3\%) and the D168A (present at a frequency of 4\%) RASs; in the other case, A156T (present at a frequency of 5\%) was detected in combination with Q80K (present at a frequency of 5\%), S122R (present at a frequency of 3\%), R155T (present at a frequency of 3\%), and D168V (present at a frequency of $8 \%$ ).

Overall, resistant variants with multiple RASs were detected at significantly higher frequencies in the liver than in serum, both when the analysis was limited to the 11 paired liver/serum samples and when the data from the 11 liver specimens were compared to all 31 serum samples investigated ( $p<0.01$ and $p=0.01$, respectively). Among serum samples from the 31 patients, 1 showed resistant variants with 5 different RASs, 5 resistant variants with 4 different RASs, 11 resistant variants with 3 RASs, 9 resistant variants with 2 different RASs, and 5 resistant variants with 1 RAS (Table 3). Among the 11 liver tissue specimens analyzed, 2 showed resistant variants with 5 different RASs, 4 resistant variants with 4 RASs, 4 resistant variants with 3 RASs, and 1 resistant variant with 2 RASs (Table 3). Overall, the detection of resistant variants with a single RAS was higher in serum samples than in liver tissues ( 0 vs. $16 \%, p=0.1$ ).

Phylogenetic analysis of NS3 sequences confirmed that all the isolates belonged to G1a, and showed that sequences clustered within 2 distinct clades, clade I and clade II [9]. In particular, 10/31 (32.2\%) patients were infected by clade I, $15 / 31(48.4 \%)$ patients were infected by clade II, and 6/31 (19.3\%) patients showed a mixed infection with both clade I and clade II NS3 sequences (Fig. 1). Of note, 4/6 individuals with mixed infections had a his- 
tory of intravenous drug abuse. As expected, the Q80K substitution was exclusively associated with clade I.

\section{Discussion}

In this study, we investigated the prevalence of baseline NS3 RASs by NGS in liver tissues and serum samples from a small number of DAAs-naïve patients from Southern Italy infected with HCV G1a. When a cutoff of $1 \%$ was applied, $100 \%$ of the analyzed patients showed at least one aa substitution related to PI resistance both in liver tissue and in serum samples. The percentage of patients with baseline NS3 RASs remained high, also when the $15 \%$ clinically relevant cutoff [4] was used, with 54.5 and $22.5 \%$ of patients harboring NS3 RASs in liver tissue specimens and serum samples, respectively. This prevalence appears to be significantly higher than that observed in previous studies [25-28]. This discrepancy might be due to differences in the assays (based on population or deep sequencing) utilized in the different studies, as well as to the choice to investigate only specific RASs while excluding others, and the increasing number of RASs over time recognized to be involved in resistance to PIs [4]. Furthermore, it has to be taken into account that the present study included the analysis of viral isolates from the liver that is the site where virus replication takes place and HCV quasispecies show the highest complexity at both nucleotide and aa levels [11, $12,14-16]$. It is known that the composition of viral population in the serum does not necessarily reflect those of the viral population in the liver. The reasons for this difference remain undefined. It has been hypothesized that the highest complexity in the liver quasispecies may be due to the presence of viruses with different degrees of fitness that are not immediately incorporated into mature virions released into the circulation. Alternatively, differences in the clearance rates or steady-state levels of some variants might be responsible for the observed discrepancy.

It is interesting to note that in the studied patients, S122R, D168V/A, and Q80K/R - all known to be involved in decreasing susceptibility also to second-generation PIs [29] - were the RASs at higher prevalence both in liver tissues and in serum samples. Though not surprising for Q80K $[5,8,30]$, this high prevalence was unexpected for $\mathrm{S} 122 \mathrm{R}$ and D168V/A. Indeed, the natural occurrence of single RASs in HCV-G1-infected patients has been reported to be lower than $3 \%$ for the majority of resistant variants to PIs [5]. This low occurrence had been linked to the impaired fitness of PI-resistant variants, as the NS3 RASs involve aa residues that are crucial for NS3 func-

NS3 Variability in Treatment-Naïve HCV-Gla Carriers tionality. An exception to this is the Q80K variant, which shows no loss of replicative fitness and has been reported as the most frequently detected NS3 substitution, albeit with important geographical differences. Indeed, Q80K is present in about half of HCV-G1a-infected patients in North America, whereas its prevalence is around 19 and 9\% in Europe and South America, respectively $[5,8,30]$. In our study population, Q80K/R was detected in 73 and $23 \%$ of cases when liver tissues and serum samples were analyzed, respectively, and it was the third most frequent substitution, even if at low frequency $(<5 \%)$ in most cases. Indeed, a single patient showed NS3 sequences harboring Q80K at a frequency higher than 15\%, and all NS3 sequences from this patient belonged to clade I. Interestingly, the 2 patients showing the Q80K RAS at a frequency higher than $5 \%$ had a mixed infection with both clade I and clade II sequences. In this context, our results from phylogenetic analysis demonstrates that both clade I and II cocirculate with an approximately similar prevalence in our geographic area - in accordance with data from other Italian and European regions [31, 32] - and that a mixed infection with both clades is quite a frequent event.

Notably, we also found that Q80K significantly associates with $\mathrm{R} 155 \mathrm{~K}$, and that this substitution in combination with Q80K could be detected at a very high frequency (up to $96 \%$ ) in the intrapatient $\mathrm{HCV}$ population, suggesting that NS3 variants showing both these RASs have good replication fitness. This is in contrast with previous data indicating that $\mathrm{R} 155 \mathrm{~K}$ in the presence of Q80K substitution negatively affects viral fitness [27]. Although in this study we did not perform quasispecies reconstruction and the different observed RASs might not be included in the same viral genome, the detection of at least one additional RAS in all the patients showing the Q80K/R155K combination tempts us to speculate that the possible additional RASs could act as compensatory substitutions rescuing viral fitness. It is noteworthy that in 2 patients we found the A156T substitution that, to our knowledge, has so far never been detected at baseline and is known to importantly affect viral fitness, and to confer resistance to newer PIs [4]. Also in these 2 patients, the poorly fitting A156T substitution was detected in possible combination with multiple additional RASs. The hypothesis of the potential compensatory effect of some substitutions might also theoretically explain the high prevalence of many of the RASs we found, although it should be underlined that in our study the combination of investigation of intrahepatic viral populations with a very sensitive NGS approach may have significantly increased the sensitivity of RAS detection compared to previous studies. Indeed, there is a fair- 
ly general agreement in considering only RASs representing more than $15 \%$ of the circulating viral populations as clinically relevant [4]. However, the arbitrary identification of this cutoff is questionable, and the hypothesis cannot be ruled out that it is the extremely high efficacy of combined DAA treatments directed against different viral targets that determines the low or null clinical impact of the many variants that, as we have shown here, are present as minor populations in HCV Gla-infected patients.

\section{Acknowledgments}

This study was supported in part by a grant from Janssen-Cilag and a grant from Gilead Sciences

\section{Disclosure Statement}

The authors of this study declare no conflicts of interest.

\section{References}

1 World Health Organization: Global hepatitis report, 2017. Geneva, WHO, 2017.

2 Liang TJ, Ghany MG: Current and future therapies for hepatitis $\mathrm{C}$ virus infection. $\mathrm{N}$ Engl J Med 2013;368:1907-1917.

3 Pawlotsky JM: Hepatitis C treatment: the data flood goes on-an update from the liver meeting 2014. Gastroenterology 2015; 148:468479.

4 Pawlotsky JM: Hepatitis C virus resistance to direct-acting antiviral drugs in interferonfree regimens. Gastroenterology 2016;151: $70-86$.

5 Sarrazin C: The importance of resistance to direct antiviral drugs in HCV infection in clinical practice. J Hepatol 2016;64:486-504.

6 Lontok E, Harrington P, Howe A, Kieffer T, Lennerstrand J, et al: Hepatitis $\mathrm{C}$ virus drug resistance-associated substitutions: state of the art summary. Hepatology 2015;62:16231632.

7 Halfon P, Locarnini S: Hepatitis C virus resistance to protease inhibitors. J Hepatol 2011; 55:192-206.

8 Sarrazin C, Lathouwers E, Peeters M, Daems B, Buelens A, et al: Prevalence of the hepatitis C virus NS3 polymorphism Q80K in genotype 1 patients in the European region. Antiviral Res 2015;116:10-16.

9 Pickett BE, Striker R, Lefkowitz EJ: Evidence for separation of HCV subtype 1a into two distinct clades. J Viral Hepat 2011;18:608618.

10 Welzel TM, Bhardwaj N, Hedskog C, Chodavarapu $\mathrm{K}$, Camus $\mathrm{G}$, et al: Global epidemiology of HCV subtypes and resistance-associated substitutions evaluated by sequencingbased subtype analyses. J Hepatol 2017;67: 224-236.

11 Bartolini B, Selleri M, Garbuglia AR, Giombini E, Taibi C, et al: HCV NS3 quasispecies in liver and plasma and dynamics of telaprevir-resistant variants in breakthrough patients assessed by UDPS: a case study. J Clin Virol 2015;72:60-65.

12 Morsica G, Andolina A, Merli M, Messina E, Hasson $\mathrm{H}$, et al: NS3 protease resistance-associated substitutions in liver tissue and plasma samples from patients infected by hepatitis $\mathrm{C}$ virus genotype 1A or 1B. Arch Virol 2017;162: 2271-2277.
13 Cubero M, Esteban JI, Otero T, Sauleda S, Bes M, Esteban R, Guardia J, Quer J: Naturally occurring NS3-protease-inhibitor resistant mutant A156T in the liver of an untreated chronic hepatitis C patient. Virology 2008;370:237245.

14 Cabot B, Martell M, Esteban JI, Sauleda S, Otero T, Esteban R, Guardia J, Gomez J: Nucleotide and amino acid complexity of hepatitis $\mathrm{C}$ vitus quasispecies in serum and liver. J Virol 2000;74:805-811.

15 Maimone S, Musolino C, Squadrito G, Raffa $\mathrm{G}$, Pollicino T, et al: NS3 genetic variability in $\mathrm{HCV}$ genotype-1b isolates from liver specimens and blood samples of treatment-naive patients with chronic hepatitis C. Antivir Ther 2013;18:131-134.

16 Maimone S, Tripodi G, Musolino C, Cacciola I, Pollicino T, et al: Lack of the NS5B S282T mutation in $\mathrm{HCV}$ isolates from liver tissue of treatment-naive patients with HCV genotype-1b infection. Antivir Ther 2015;20:245247.

17 Fourati S, Pawlotsky JM: Virologic tools for HCV drug resistance testing. Viruses 2015;7: 6346-6359.

18 Kalaghatgi P, Sikorski AM, Knops E, Rupp D, Sierra S, et al: Geno2pheno $[\mathrm{HCV}]-$ a webbased interpretation system to support hepatitis $C$ treatment decisions in the era of directacting antiviral agents. PLoS One 2016; 11:e0155869.

19 Bolger AM, Lohse M, Usadel B: Trimmomatic: a flexible trimmer for Illumina sequence data. Bioinformatics 2014;30:2114-2120.

20 Li H, Durbin R: Fast and accurate short read alignment with Burrows-Wheeler transform. Bioinformatics 2009;25:1754-1760.

21 Camacho C, Coulouris G, Avagyan V, Ma N, Papadopoulos J, et al: BLAST+: architecture and applications. BMC Bioinformatics 2009; 10:421.

22 Ruggieri V, Anzar I, Paytuvi A, Calafiore R, Cigliano RA, et al: Exploiting the great potential of sequence capture data by a new tool, SUPER-CAP. DNA Res 2017;24:81-91.

23 Li H, Handsaker B, Wysoker A, Fennell T, Ruan J, et al: The Sequence Alignment/Map format and SAMtools. Bioinformatics 2009; 25:2078-2079.
24 Kumar S, Stecher G, Tamura K: MEGA7: Molecular Evolutionary Genetics Analysis version 7.0 for bigger datasets. Mol Biol Evol 2016;33:1870-1874.

25 Kuntzen T, Timm J, Berical A, Lennon N, Berlin AM, et al: Naturally occurring dominant resistance mutations to hepatitis $C$ virus protease and polymerase inhibitors in treatment-naive patients. Hepatology 2008; 48: 1769-1778.

26 Sarrazin C, Dvory-Sobol H, Svarovskaia ES, Doehle BP, Pang PS, et al: Prevalence of resistance-associated substitutions in HCV NS5A, NS5B, or NS3 and outcomes of treatment with ledipasvir and sofosbuvir. Gastroenterology 2016;151:501-512 e501.

27 Lenz O, Verbinnen T, Fevery B, Tambuyzer L, Vijgen L, et al: Virology analyses of HCV isolates from genotype 1 -infected patients treated with simeprevir plus peginterferon/ribavirin in phase IIb/III studies. J Hepatol 2015;62: 1008-1014.

28 Larrat S, Vallet S, David-Tchouda S, Caporossi A, Margier J, et al: Naturally occurring resistance-associated variants of hepatitis C virus protease inhibitors in poor responders to pegylated interferon-ribavirin. J Clin Microbiol 2015;53:2195-2202.

29 Zeuzem S, Ghalib R, Reddy KR, Pockros PJ, Ben Ari Z, et al: Grazoprevir-elbasvir combination therapy for treatment-naive cirrhotic and noncirrhotic patients with chronic hepatitis $C$ virus genotype 1,4 , or 6 infection: a randomized trial. Ann Intern Med 2015;163: $1-13$.

30 McCloskey RM, Liang RH, Joy JB, Krajden M, Montaner JS, et al: Global origin and transmission of hepatitis $\mathrm{C}$ virus nonstructural protein 3 Q80K polymorphism. J Infect Dis 2015;211:1288-1295.

31 De Luca A, Di Giambenedetto S, Lo Presti A, Sierra S, Prosperi M, et al: Two distinct hepatitis $\mathrm{C}$ virus genotype 1a clades have different geographical distribution and association with natural resistance to NS3 protease inhibitors. Open Forum Infect Dis 2015;2:ofv043.

32 Santos AF, Bello G, Vidal LL, Souza SL, Mir $\mathrm{D}$, et al: In-depth phylogenetic analysis of hepatitis $C$ virus subtype la and occurrence of $80 \mathrm{~K}$ and associated polymorphisms in the NS3 protease. Sci Rep 2016;6:31780. 